\title{
Human Borrelia miyamotoi Infection, Austria
}

\author{
Selma Tobudic, Heinz Burgmann Gerold Stanek, Stefan Winkler, \\ Anna-Margarita Schötta, Markus Obermüller, Mateusz Markowicz, Heimo Lagler
}

We report a human case of Borrelia miyamotoi infection diagnosed in Austria. Spirochetes were detected in Giemsa-stained blood smears. The presence of $B$. miyamotoi in the patient's blood was confirmed by PCR, and phylogenetic analysis identified an infection with a strain from Europe.

$B$ orrelia miyamotoi is a relapsing fever spirochete transmitted by the same genus of ticks that transmits B. burgdorferi sensu lato (s.1.), Anaplasma phagocytophilum, Babesia species, and tickborne flaviviruses (1-3). B. miyamotoi has been documented in ticks from the United States and in numerous countries in Europe (including Russia), as well as in Japan (1,4-6). B. miyamotoi also has been found in Ixodes scapularis ticks in the northeastern and north-central United States and adjoining areas of Canada, in I. pacificus ticks in the far western United States and British Columbia, in I. ricinus ticks in Europe, and in I. persulcatus ticks in Europe and Asia $(1,7,8)$. I. pavlovskyi and I. ovatus ticks in northern Asia are 2 other species that have been shown to carry B. miyamotoi (9). Endemic areas of B. miyamotoi in Ixodes ticks overlap with those of B. burgdorferi s.l. but with 10-fold lower prevalence (4). Co-infection of Ixodes ticks with both spirochetes also has been identified (9).

Unlike Lyme borreliosis, patients with B. miyamotoi disease typically do not have skin lesions but instead have a nonspecific febrile illness, potentially associated with leukopenia, thrombocytopenia, and elevated liver function parameters (10). Highly immunocompromised patients might have chronic meningitis (2). Untreated patients with B. miyamotoi disease might experience a limited number of recurrent

Author affiliations: Division of Infectious Diseases and Tropical Medicine, Medical University of Vienna, Vienna, Austria

(S. Tobudic, H. Burgmann S. Winkler, M. Obermüller, H. Lagler); Institute for Hygiene and Applied Immunology, Center for Pathophysiology, Infectiology and Immunology, Medical University of Vienna, Vienna (G. Stanek, A.-M. Schötta, M. Markowicz)

DOI: https://doi.org/10.3201/eid2609.191501 episodes of fever, similar to other relapsing fevers caused by Borrelia infections (6). The same antibiotic regimens used to treat Lyme borreliosis (e.g., 10-14day courses of oral doxycycline or amoxicillin) are effective for B. miyamotoi disease. Parenteral therapy with ceftriaxone would be preferred for patients with meningitis (3).

Diagnosis of B. miyamotoi disease should be considered in any patient who has fever attacks and resides in or has spent time during tick season in a region where Lyme borreliosis is endemic. Diagnosis requires confirmation using PCR. If the density of spirochetes in the blood is $\geq 10^{4} / \mathrm{mL}$, spirochetes might be identified by examining several high-power fields of the blood smear or centifuged sample of cerebrospinal fluid stained with Giemsa or Wright stain (10). Several PCR assays can detect B. miyamotoi in whole blood, plasma, and cerebrospinal fluid by using primers specific for $16 \mathrm{~S}$ ribosomal RNA and for the flaB and $g l p Q$ genes $(2,3,8)$. Serologic testing based on glycerophosphodiester phosphodiesterase antigen of B. miyamotoi that is not found in B. burgdorferi s.l. is highly sensitive but only on convalescent-phase serum specimens (11).

\section{The Case-Patient}

A 51-year-old woman who had a long medical history of seropositive rheumatoid arthritis treated with rituximab sought care at our outpatient clinic for relapsing fever that started 3 months before. Fever episodes occurred every 5 days, and duration ranged from 2 to 3 days. Four weeks before the onset of symptoms, the patient had returned from a 3-week trip through the United States, where, as a tourist, she visited the East and West Coasts and stayed in several national parks. She reported several insect bites and 1 tick bite without erythema migrans that occurred while she was in the United States but did not notice any tick bites before or after her travel. After her return, the patient did not travel abroad again but spent her time in her home in lower Austria. 
Doxycline $200 \mathrm{mg}$ once daily for 10 days

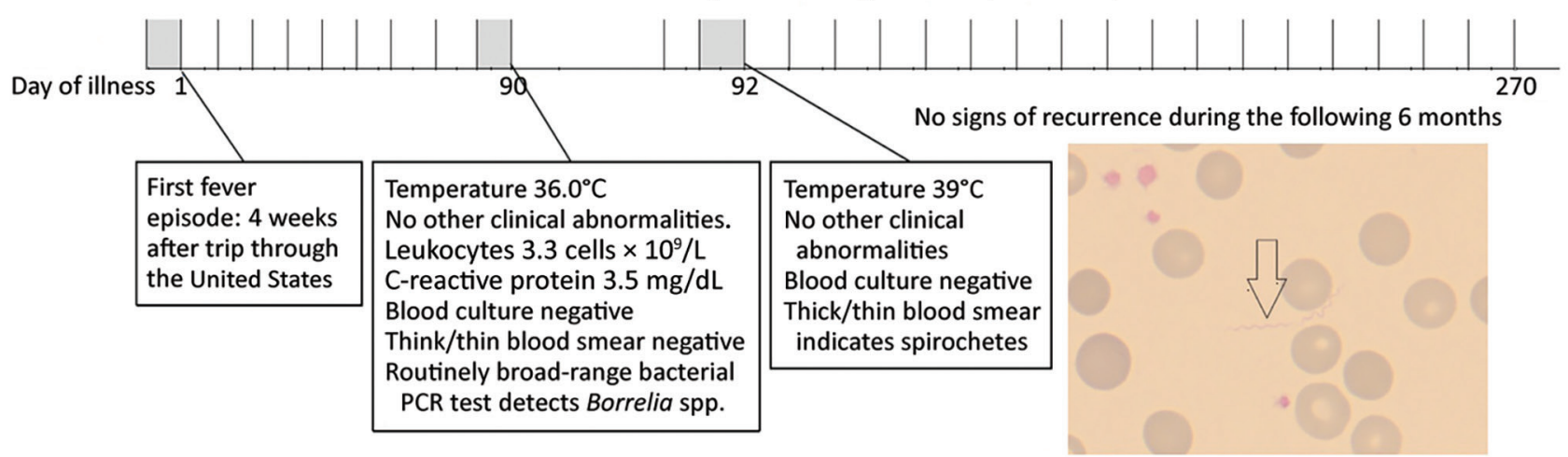

Figure 1. Timeline of the course of symptoms and treatment, including laboratory test results, for a patient with Borrelia miyamotoi infection (including Giemsa stain of thin blood smear on day 92), Austria. Arrows indicate spirochetes. Original magnification $\times 100$.

Figure 2. Phylogenetic tree based on the 16S rRNA gene of Borrelia miyamotoi from a patient in Austria (Bm4667; arrow) and reference sequences. This phylogenetic tree was constructed by using the maximum-likelihood method based on the general time reversible model. The tree is drawn to scale, with bootstrap values shown at the nodes of the tree, inferred from 600 replicates. A total of $1,199 \mathrm{bp}$ of B. miyamotoi $16 \mathrm{~S}$ rRNA gene sequences was used in the final dataset, which involved $34 \mathrm{nt}$ sequences and in which gaps and missing data were deleted. Evolutionary analyses were conducted in MEGA7 (https://www.megasoftware. net). The patient isolate clustered together with strains found in ticks from Europe. Information of the source and country for reference isolates is shown in parentheses after strain designation. Scale bar indicates substitutions per site.
KU749373.1 Borrelia miyamotoi strain 13T392 (China, tick)

KU749375.1 Borrelia miyamotoi strain 14T52 (China, tick)

KU749372.1 Borrelia miyamotoi strain 15H32 (China, tick)

JF951379.1 Borrelia miyamotoi isolate Kurgan-09(3)-IP (Russia, tick)

GQ253925.1 Borrelia miyamotoi strain De1 (Russia, human)

EF488992.1 Borrelia sp. 57Nsk (Russia, tick)

CP024390.1 Borrelia miyamotoi strain Izh-4 (Russia, human)

CP024371.1 Borrelia miyamotoi strain Izh-14 (Russia, human)

CP024351.1 Borrelia miyamotoi strain Izh-16 (Russia, human)

CP024333.1 Borrelia miyamotoi strain Yekat-1 (Russia, human)

CP024316.1 Borrelia miyamotoi strain Yekat-6 (Russia, human)

CP024205.1 Borrelia miyamotoi strain Izh-5 (Russia, human)

CP004217.2 Borrelia miyamotoi FR64b (Japan, rodent or tick)

AY604676.1 Borrelia miyamotoi isolate FR64b (USA, tick)

AB904793.1 Borrelia miyamotoi HT31 (Japan, tick)

AB900817.1 Borrelia miyamotoi strain Y13T1 (Japan, tick)

KX769848.1 Borrelia miyamotoi isolate Khekhtzir_2011-34 (Russia, tick)

- KU749374.1 Borrelia miyamotoi strain 15H609 (China, human)

- KU749376.1 Borrelia miyamotoi strain 14T114 (China, tick)

— NR_025861.1 Borrelia miyamotoi strain HT 31 (Japan, tick)

LC064032.1 Borrelia miyamotoi (Russia, tick)

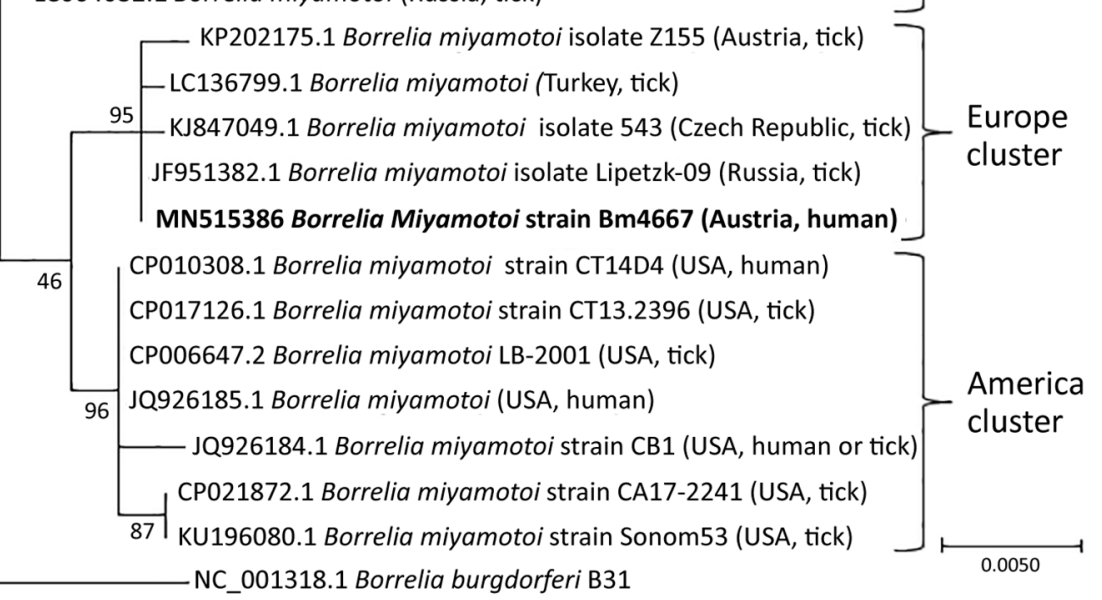


No abnormal findings were observed on physical examination; in particular, no apparent rash on the skin was noted. Routine laboratory tests performed were normal (including kidney and liver function tests), except for the evidence of leukopenia with 3.3 $\times 10^{9}$ cells/L (reference range 4-10 $\times 10^{9}$ cells/L) and slightly elevated C-reactive protein of $3.5 \mathrm{mg} / \mathrm{dL}$ (reference range $<0.5 \mathrm{mg} / \mathrm{dL}$ ). Multiple blood and urine cultures were negative (Figure 1). An initial peripheral Giemsa-stained blood smear was performed during an afebrile period without any result, but when the test was repeated during the next fever episode, spirochetes were detected between blood cells (Figure $1)$. Detection of spirochetes in blood smear, which is not typical in cases of $B$. miyamotoi infection, could be attributable to prolonged spirochetemia likely associated with rituximab therapy. Borrelia spp. was identified in a routinely broad-range bacterial 165 genebased PCR test (SepsiTest-UMD; Molzym GmbH \& Co. KG, https://www.molzym.com). DNA was extracted from EDTA blood, and a real-time PCR assay specific for B. miyamotoi targeting the $g l p Q$ gene (5) was positive. To confirm the genospecies present in the patient's blood, further PCRs targeting the 16S$23 \mathrm{~S}$ internal transcribed spacer region $(12,13), 16 \mathrm{~S}$ rRNA, and $g l p Q$ gene $(14,15)$ were performed, and amplicons were sent to a laboratory for bidirectional sequencing (Microsynth, Vienna, Austria). All PCRs confirmed the presence of $B$. miyamotoi in the patient's blood, and all yielded $100 \%$ identity to various B. miyamotoi strains in the GenBank database. Using MEGA7 (https://www.megasoftware.net), we constructed a phylogenetic tree for our isolate $\mathrm{Bm} 4667$ on the basis of the obtained 16S rRNA gene sequence (Figure 2). All 3 sequences obtained during this investigation were submitted to GenBank (accession no. MN515386 for the 16S rRNA gene, MT396940 for the $g l p Q$ gene, and MT396941 for the 16S-23S internal transcribed spacer region).

The patient was treated with $200 \mathrm{mg}$ doxycycline once daily for 2 weeks. On the first day of antibiotic administration, the patient was admitted to our hospital for observation in case of potential Jarisch-Herxheimer reaction. However, during the therapy, no reaction or adverse effects were detected. The patient recovered successfully, and no signs of recurrence were observed in the following 6 months.

\section{Conclusions}

We describe a human case of $B$. miyamotoi infection diagnosed in Austria. Although the patient's report of a tick bite in the United States suggested that this infection was an imported case, the phylogenetic analysis of the B. miyamotoi strain clearly indicates an infection with a strain from Europe (and possibly Austria). Clinicians should be aware of the possibility of these infections.

\section{Acknowledgment}

We thank the patient for providing consent to publish information about her case.

\section{About the Author}

Dr. Tobudic is an internal medicine specialist at the Department of Infection Diseases and Tropical Medicine, Medical University of Vienna. Her research interest includes yeast infections, biofilm research, pathomechanism of antimicrobial resistance, and research of tickborne illness.

\section{References}

1. Fukunaga M, Takahashi Y, Tsuruta $Y$, Matsushita O, Ralph D, McClelland M, et al. Genetic and phenotypic analysis of Borrelia miyamotoi sp. nov., isolated from the ixodid tick Ixodes persulcatus, the vector for Lyme disease in Japan. Int J Syst Bacteriol. 1995;45:804-10. https:/ / doi.org/ 10.1099/00207713-45-4-804

2. Gugliotta JL, Goethert HK, Berardi VP, Telford SR III. Meningoencephalitis from Borrelia miyamotoi in an immunocompromised patient. N Engl J Med. 2013;368:240-5. https://doi.org/10.1056/NEJMoa1209039

3. Hovius JW, de Wever B, Sohne M, Brouwer MC, Coumou J, Wagemakers A, et al. A case of meningoencephalitis by the relapsing fever spirochaete Borrelia miyamotoi in Europe. Lancet. 2013;382:658. https:/ / doi.org/10.1016/ S0140-6736(13)61644-X

4. Crowder CD, Carolan HE, Rounds MA, Honig V, Mothes B, Haag $\mathrm{H}$, et al. Prevalence of Borrelia miyamotoi in Ixodes ticks in Europe and the United States. Emerg Infect Dis. 2014;20:1678-82. https://doi.org/10.3201/eid2010.131583

5. Reiter M, Schötta AM, Müller A, Stockinger H, Stanek G. A newly established real-time PCR for detection of Borrelia miyamotoi in Ixodes ricinus ticks. Ticks Tick Borne Dis. 2015;6:303-8. https:/ / doi.org/10.1016/j.ttbdis.2015.02.002

6. Platonov AE, Karan LS, Kolyasnikova NM, Makhneva NA, Toporkova MG, Maleev VV, et al. Humans infected with relapsing fever spirochete Borrelia miyamotoi, Russia. Emerg Infect Dis. 2011;17:1816-23. https:// doi.org/10.3201/ eid1710.101474

7. Scoles GA, Papero M, Beati L, Fish D. A relapsing fever group spirochete transmitted by Ixodes scapularis ticks. Vector Borne Zoonotic Dis. 2001;1:21-34. https:/ / doi.org/ 10.1089/153036601750137624

8. Richter D, Schlee DB, Matuschka FR. Relapsing fever-like spirochetes infecting European vector tick of Lyme disease agent. Emerg Infect Dis. 2003;9:697-701. https:/ / doi.org/ 10.3201/eid0906.020459

9. Takano A, Toyomane K, Konnai S, Ohashi K, Nakao M, Ito T, et al. Tick surveillance for relapsing fever spirochete Borrelia miyamotoi in Hokkaido, Japan. PLoS One. 2014;9:e104532. https:// doi.org/10.1371/journal.pone.0104532

10. Krause PJ, Fish D, Narasimhan S, Barbour AG. Borrelia miyamotoi infection in nature and in humans. Clin Microbiol Infect. 2015;21:631-9. https://doi.org/10.1016/ j.cmi.2015.02.006 
11. Schwan TG, Schrumpf ME, Hinnebusch BJ, Anderson DE Jr, Konkel ME. GlpQ: an antigen for serological discrimination between relapsing fever and Lyme borreliosis. J Clin Microbiol. 1996;34:2483-92. https:/ / doi.org/10.1128/ JCM.34.10.2483-2492.1996

12. Bunikis J, Garpmo U, Tsao J, Berglund J, Fish D, Barbour AG.Sequence typing reveals extensive strain diversity of the Lyme borreliosis agents Borrelia burgdorferi in North America and Borrelia afzelii in Europe. Microbiology. 2004;150:1741-55. https://doi.org/10.1099/ mic.0.26944-0

13. Wilhelmsson P, Fryland L, Börjesson S, Nordgren J, Bergström S, Ernerudh J, et al. Prevalence and diversity of Borrelia species in ticks that have bitten humans in Sweden. J Clin Microbiol. 2010;48:4169-76. https:/ / doi.org/10.1128/ JCM.01061-10
14. Fomenko NV, Livanova NN, Borgoiakov VI, Kozlova IV, Shula1kina IV, Pukhovskaia NM, et al. Detection of Borrelia miyamotoi in ticks Ixodes persulcatus from Russia [in Russian]. Parazitologiia. 2010;44:201-11.

15. Fukunaga M, Hamase A, Okada $K$, Inoue $H$, Tsuruta $Y$, Miyamoto K, et al. Characterization of spirochetes isolated from ticks (Ixodes tanuki, Ixodes turdus, and Ixodes columnae) and comparison of the sequences with those of Borrelia burgdorferi sensu lato strains. Appl Environ Microbiol. 1996;62:2338-44. https:// doi.org/10.1128/AEM.62.7.2338-2344.1996

Address for correspondence: Selma Tobudic, Department of Internal Medicine I, Division of Infectious Diseases and Tropical Medicine, Währinger Gürtel 18-20, A-1090 Vienna, Austria; email: selma.tobudic@meduniwien.ac.at

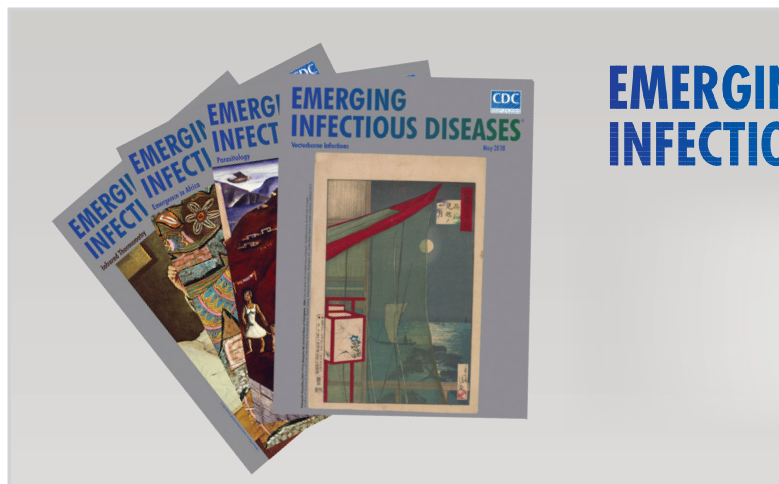

- History of Mosquitoborne Diseases in the United States and Implications for New Pathogens

- Surveillance for Mosquitoborne Transmission of Zika Virus, New York City, NY, USA, 2016

- Two Cases of Israeli Spotted Fever with Purpura Fulminans, Sharon District, Israel

- Antimicrobial Resistance in Invasive Bacterial Infections in Hospitalized Children, Cambodia, 2007-2016

- Epidemic Dynamics of Vibrio parahaemolyticus Illness in a Hotspot of Disease Emergence, Galicia, Spain

- Dynamics of Spirochetemia and Early PCR Detection of Borrelia miyamotoi

- Transmission of Severe Fever with Thrombocytopenia Syndrome Virus by Haemaphysalis longicornis Ticks, China

- Seroprevalence of Severe Fever with Thrombocytopenia Syndrome Virus Antibodies in Rural Areas, South Korea

- Human Usutu Virus Infection with Atypical Neurologic Presentation, Montpellier, France, 2016

- Spread of Plague by Respiratory Droplets or Ectoparasites
- Alkhurma Hemorrhagic Fever Virus RNA in Hyalomma rufipes Ticks Infesting Migratory Birds, Europe and Asia Minor

- Cholera Epidemic in South Sudan and Uganda and Need for International Collaboration in Cholera Control

- External Quality Assessment for Zika Virus Molecular Diagnostic Testing, Brazil

- A Mental Models Approach to Assessing Public Understanding of Zika Virus, Guatemala

- Heartland Virus and Hemophagocytic Lymphohistiocytosis in Immunocompromised Patient, Missouri, USA

- Equine Encephalosis Virus in India, 2008 Epizootic Hemorrhagic Disease Virus Serotype 6 Infection in Cattle, Japan, 2015

- Fatal Visceral Leishmaniasis Caused by Leishmania infantum, Lebanon

- Second Human Pegivirus in Hepatitis C Virus-Infected and Hepatitis C Virus/HIV-1-Co-infected Persons Who Inject Drugs, China

- Characterization of Clinical Isolates of Bartonella henselae Strains, South Korea 